\title{
Comparison of Reaction Time Between eSports Players of Different Genres and Sportsmen
}

\author{
Peter Bickmann, German Sport University Cologne, Germany \\ Konstantin Wechsler, German Sport University Cologne, Germany \\ Kevin Rudolf, German Sport University Cologne, Germany \\ (iD https://orcid.org/0000-0001-9815-3475 \\ Chuck Tholl, German Sport University Cologne, Germany \\ Ingo Froböse, German Sport University Cologne, Germany \\ Christopher Grieben, German Sport University Cologne, Germany
}

\begin{abstract}
Quick reactions are considered important in both traditional and electronic sports, and research findings suggest that reaction time can be optimized by both sports activity and playing action video games. In this study, reaction and motor times of 18 professional and 21 non-professional eSports players from different genres and 36 non-professional traditional sportsmen were compared using the Vienna Test System. No differences between the groups were found in simple visual, acoustic, and choice reaction times. Differentiated by game genre, players from sports simulations had significantly shorter reaction times than MOBA players in the acoustic and choice reaction test. The results of this study suggest that traditional sports and eSports may improve reaction times to a similar amount. Furthermore, various game genres require different reaction times or may affect related abilities in different ways.
\end{abstract}

\section{KEYWORDS}

Acoustic Reaction, Athletes, Choice Reaction, eSports, Motor Time, Professional Players, Reaction Time, Traditional Sports, Visual Reaction

\section{INTRODUCTION}

Electronic sports (eSports) represents a growing market while a large number of people invest considerable time playing video games. With an estimated revenue of $\$ 1.1$ billion and an audience up to 495 million in 2020, the business continues to grow (Newzoo, 2020). The competition for victories is also becoming increasingly intense as the number of players and prize money increases. Professional and non-professional gamers compete in different game genres such as multiplayer online battle arena (MOBA), real time strategy (RTS), first-person shooter (FPS), Fighting Games, or sports simulations (Bányai et al., 2019). Most of these eSports titles require high speed reactions. The players are required to react to different visual and acoustic stimuli before making the correct decision as quickly as possible (Kowal et al., 2018). Acting quickly seems to be important, because responding incorrectly or too 
slowly can lead to defeat in a matter of seconds. The importance of being fast is also shown by the fact that gamers outperform non-gamers in terms of simple reaction times (Richardson et al., 2014). Nevertheless, little is known about performance parameters of the players or how to improve them (Green, 2018; Pedraza-Ramirez et al., 2020). On the other hand, in traditional sports, research into performance parameters has already progressed further. Just as in eSports, fast reaction times can be a decisive factor (Dykiert et al., 2012) and are significant for better performance of athletes (Ghuntla et al., 2014) for instance in short sprints (Paradisis, 2013). Additionally, perceptual and attentional skills are important for performance in sports-related tasks like quick decision-making (Hüttermann et al., 2019). Therefore, it comes to no surprise that sportspeople also showed significantly shorter reaction times than non-sportspeople in numerous studies (Atan \& Akyol, 2014). Thus, similar to eSports, traditional sports can positively affect one's reaction times and vice versa.

Various studies have already compared reaction times of gamers and non-gamers or sportspeople and non-sportspeople (e.g., Atan \& Akyol, 2014; Richardson et al., 2014), but none of them actually compared gamers and sportspeople. Therefore, this study compares reaction times of participants from both traditional and electronic sports. In contrast to most studies, which divided gamers according to their videogame playing time, participant selection in the present study was based on the professional level of the players. Simple visual, acoustic, and choice reaction times of nonprofessional traditional sportspeople (TS), professional (PP), and non-professional eSports players (NPP) were measured in three different tests. In a further analysis, the eSports players' reaction times were additionally compared regarding their game genre. Comparable studies, such as Li et al. (2020), have more participants from a single game genre (MOBA). This study, on the other hand, compares different eSports players and traditional sportspeople in a variety of areas and thereby serves as a pilot study. Since the number of participants especially in the different game genres was quite small, these results should be interpreted as first insights into this research area. For future work, results from this study should be used to compare targeted abilities like reaction time of eSports players or traditional sportspeople of specific areas.

\section{BACKGROUND}

In the context of this study, eSports is defined as competitive gaming. Players train and improve mental and physical abilities to improve their chances of success against other players on electronic devices such as computers or gaming consoles (Wagner, 2006). Competition in eSports is often implemented in tournaments, but can also take place within a game, for instance through a ladder system. In recent years, the scientific acceptance of eSports has also increased in different fields of research. The subjects investigated include the eSports market and sponsoring (Mangeloja, 2019), consumption motives (Bányai et al., 2019), motivational and participation factors (Braun et al., 2016), or the competition of eSports (Lipovaya et al., 2018). Research was also conducted on the characteristics of eSports players and essential skills for high performance. Along with in-game skills, for instance a sound knowledge of the game or "mechanical skills" (Donaldson, 2015), eSports players require high cognitive skills (Happonen \& Minashkina, 2019; Himmelstein et al., 2017), to meet the games' requirements, for instance quick decision making or high attentional skills, in fast paced eSport titles (Voss et al., 2010).

The actual field of game performance in eSports titles, however is relatively unexplored (PedrazaRamirez et al., 2020). Depending on the game genre, there are several factors that can give information on the players' performance, such as the kill/death ration in FPS games or gold per minute and kill/ death ratio in MOBA games (Parshakov et al., 2018). But these parameters are linked to several problems. Similar to traditional sports, the own performance in competitions always depends on the opponent's performance. A parameter like shooting power in soccer, which can be measured without the influence of the opponent, does not exist in eSports. Additionally, in multiple eSports titles such as MOBAs, players take on different roles with different tasks. In soccer, the performance of a defender 
is rarely measured by the goals scored. Similarly, a supporting player in a MOBA may have a worse kill/death ratio than another player, but still performed well (Hodge et al., 2019). For this reason, other performance factors, for instance cognitive skills, have to be investigated to get insights into a player's actual performance capacity. Research testing cognitive performance on laboratory settings could reveal more about the eSports players' cognitive performance (Pedraza-Ramirez et al., 2020).

A possible performance indicator tested in this study is the ability to react quickly to different stimuli. Recent studies were able to show that gamers outperform non-gamers in terms of simple reaction times (Richardson et al., 2014). These results are consistent with the results of other studies, in which especially playing action video games (like FPS) can improve various cognitive functions (Green \& Bavelier, 2003; Schubert et al., 2015), for instance attentional (Schubert et al., 2015), (visual-)spatial (Feng et al., 2007), perceptual (Green et al., 2010), and perceptual-motor skills (Chen et al., 2015).

However, there are hardly any studies that compare eSports players in terms of their performance level or their game genre. Therefore, in this study, reaction times of differently skilled eSports players are compared to gain further knowledge on individual performance indicators. Since quick reaction times are of great importance for success in most fast-paced eSports titles, it is assumed that professional players outperform non-professional eSports players in reaction tests. Additionally, reaction times of sportspeople were tested and compared to the gamers' results. Furthermore, research has shown that sports also positively influences one's reaction time, while it is still assumed that professional players show quicker reaction times than sportspeople. These results are also compared with a standard sample provided by the manufacturer of the test battery. In order to make further statements about the performance profile of eSports players in different game genres, the results of these players were additionally compared dependent on their specific game genre.

\section{METHOD}

\section{Participants}

For the present cross-sectional study, 36 non-professional sportspeople (19 females; $22.75 \pm 3.25$ years) from different sports and 39 eSport players ( 3 females, $23.38 \pm 3.51$ years) were recruited via bulletins at the website of German Sport University Cologne and through personal contacts. The nonprofessional sportspeople followed their sports routine regularly and several times per week. In order to avoid possible influence of both sports and eSports on the reaction times, traditional sportspeople were not allowed to play videogames regularly. A frequency of five times a month, which is roughly equivalent to no more than once a week, was set as the maximum value. For this reason, the data of two traditional sportspeople were excluded. The 39 eSports players were classified as professional eSports players ( $n=18 ; 1$ female, $22.83 \pm 3.11$ years) if they earn money as main income by playing their game competitively, or as non-professional eSports players ( $n=21,2$ females, $23.86 \pm 3.83$ years), who also play their game competitively in tournaments, but do not earn noteworthy prize money.

All participants reported to be physically and mentally healthy and none of them suffered from limitations in the acoustic perception (all self-report). Blind spot diagnosis was carried out using Multifixation Campimetry On Line software (Damato \& Groenwald, 2003) and the EyesCream Software II (EyesAge.org, 2014). The participants were able to wear glasses or contact lenses during the entire study if needed. They provided written informed consent. The study was approved by the ethical committee of the German Sport University Cologne (reference: 053/2018).

\section{Materials}

For the reaction tests, the Vienna Test System (VTS) (Schuhfried \& Prieler, 1997) was used. The system measures different aspects of reaction behavior and different response types. For this setup, the participants had to react in three different tests: 
- $\quad$ RT1: Simple visual reaction test

- RT2: Simple acoustic reaction test

- RT3: Choice reaction test

The operating tool was the advanced response panel of the VTS, which was connected to a notebook. A rest key and a reaction key enabled the distinction between pure reaction time, which was gathered from the start of the stimulus presentation until the finger lift-off, and the motor reaction time measured from finger lift-off until the pressing of the reaction key. Visual stimuli were presented on an external 24 -inch screen. For the acoustic stimuli participants were wearing headphones. The volume was freely adjustable.

\section{Design}

Before the tests, all participants filled out questionnaires on their gaming and movement behavior throughout the last six months and their physical and mental condition. The eSports players answered questions on their gaming experience in years, and their average game time per week within the last six weeks. They were also asked about their average duration of sports per week within the last six weeks. Traditional sportsman answered questions about the time they had been exercising regularly, the average duration of sports per week, and their main sports. They were also asked about the average of how many days a month they had played computer games in the last six months. If an athlete was regularly playing computer games, he or she was excluded from the study. In addition, all participants answered questions about their physical and mental health, which were subsequently checked by the test leader.

The order of the reaction tests was identical for each participant. Each one started with the simple visual reaction test (RT1), continued with the simple acoustic reaction test (RT2) and ended with the choice reaction test (RT3). Before each test, participants completed a short practice trial. Participants were allowed to use their preferred hand and only one finger (they all used the index finger). The finger was placed on a golden rest key. When a reaction was required, participants pressed a wide black reaction key as fast as possible and returned to the rest key.

The stimulus for the simple visual reaction test was a black dot that was presented on the screen. When the dot switched its color to yellow, participants had to push the reaction key. In the simple acoustic reaction test, the stimulus was an acoustic beep signal. For the visual and acoustic test, the number of correct responses, reaction times and motor reaction times were registered. Additionally, trials in which a participant either did not react at all or not completely, i.e., they left the rest key but missed the reaction key, were registered. In both tasks, 28 stimuli were presented.

The stimulus for the choice reaction test was a yellow and a red dot or a yellow dot and an auditory stimulus, which were both presented simultaneously. No response was allowed for any other combination. The number of correct responses, reaction times, motor reaction times, and false responses were registered. A total of 50 stimuli were presented in the choice reaction test, of which 16 were relevant and required a response.

\section{Data Processing}

In order to process the extracted results and data, outliers within the data of every single participant were identified by the \pm 3.29 standard deviation criterion (Tabachnick \& Fidell, 2010) and excluded. For instance, data in the visual reaction test trials was excluded if the single reaction time differed significantly (more than 3.29 standard deviations) from the average reaction time of the participant in this test. One reason for this could be a blink or a brief moment of inattention. Moreover, data from the three groups was pooled within and the same procedure was applied to the entire data of the separate groups. For instance, one participant's choice reaction test was removed from the data because it was incorrectly responded in more than half of all trials. Since this participant seemed to not fully understand the test despite thorough explanation, and the number of incorrect responses 
Table 1. Participating professional (PP) and non-professional players' (NPP) descriptive data

\begin{tabular}{|l|l|l|l|l|l|l|l|}
\hline & Age (years) & $\begin{array}{l}\text { Gaming } \\
\text { experience } \\
\text { (years) }\end{array}$ & $\begin{array}{l}\text { Game time } \\
\text { (h)/Week }\end{array}$ & $\begin{array}{l}\text { Sports (h) / } \\
\text { Week }\end{array}$ & MOBA & FPS & $\begin{array}{l}\text { Sports } \\
\text { simulation }\end{array}$ \\
\hline PP (18) & $22.83 \pm 3.11$ & $\begin{array}{l}12.61 \pm \\
4.38\end{array}$ & $\begin{array}{l}36.69 \pm \\
13.68^{*}\end{array}$ & $4.17 \pm 3.67$ & 4 & 1 & 13 \\
\hline NPP (21) & $23.86 \pm 3.83$ & $\begin{array}{l}10.69 \pm \\
5.37\end{array}$ & $\begin{array}{l}23.19 \pm \\
12.08^{*}\end{array}$ & $4.81 \pm 5.19$ & 11 & 5 & 5 \\
\hline
\end{tabular}

${ }^{*} p<.05$

differed significantly (more than 3.29 standard deviations) from the average number of errors in the group, this data set was removed to no distort the group result in the quite small sample size.

All data was analyzed with the software IBM SPSS Statistics 26 (IBM, 2019). Statistical differences between the three groups were studied using ANOVA, Welch's t-test, and Bonferroni post hoc test. Differences between the performances within a group were analyzed using a t-test for paired samples. Pearson's test was used for correlations (medium effects for $r>.30$; large effects for $\mathrm{r}>.50)$. The significance level for all analyses was set at $\mathrm{p}<.05$.

Because there were significantly more female participants in the sportspeople group compared to the other two, all tests were analyzed including and excluding female participants, in case there were differences related to the gender. Results were tested for significant differences between male and female participants within the three groups, as well as for differences between the three groups including and excluding female participants. None of the tests indicated that male and female participants differed in terms of the measured reaction times. Therefore, female participants were not excluded in statistical analysis to strengthen the power of the data through a larger number of participants.

\section{RESULTS}

\section{Descriptive Data}

The following paragraphs provide an overview of the participants' descriptive data. Table 1 represents the descriptive data for the professional and non-professional eSports players.

The data for the game time and traditional sports per week refer to the last six weeks. Professional players played significantly more hours per week than non-professional players $(\mathrm{t}(37)=-3.27 ; \mathrm{p}<.05)$. Most professional players played the sports simulations FIFA 19 (EA Vancouver, 2018), whereas most non-professional players played the MOBA League of Legends (Riot Games, 2009).

Table 2 represents the descriptive data for the traditional sportspeople.

Sports experience is defined as the period since the participants started doing sports regularly. Data for duration of sports per week refer to the last six weeks. The represented sports are soccer, basketball, and handball for team sports and running and swimming for endurance sports. There was one participant, who engaged in horse riding and one in gymnastics (others).

\section{Differences Between ESports Players of Different Performance Levels and Sportspeople}

Table 3 represents data for all three groups in the simple visual reaction test.

The groups differed statistically significant in correct responses $(F(2,70)=3.97, p<.05, f=.10)$. A post hoc test revealed that professional players showed significantly more correct responses than non-professional players $(\mathrm{p}<.05)$, however, not more than traditional sportspeople $(\mathrm{p}=.14)$. Reaction and motor reaction times did not differ significantly. 
Table 2. Non-professional traditional sportspeople (TS) descriptive data

\begin{tabular}{|l|l|l|l|l|l|l|l|l|}
\hline & Age (years) & $\begin{array}{l}\text { Sports } \\
\text { experience } \\
\text { (years) }\end{array}$ & $\begin{array}{l}\text { Sports } \\
\text { (h) / } \\
\text { Week }\end{array}$ & $\begin{array}{l}\text { Team } \\
\text { and ball } \\
\text { sports }\end{array}$ & $\begin{array}{l}\text { Endurance } \\
\text { sports }\end{array}$ & $\begin{array}{l}\text { Fitness } \\
\text { training }\end{array}$ & $\begin{array}{l}\text { Combat } \\
\text { sports }\end{array}$ & Others \\
\hline TS (36) & $22.75 \pm 3.25$ & $\begin{array}{l}12.47 \pm \\
4.43\end{array}$ & $\begin{array}{l}9.31 \pm \\
4.22\end{array}$ & 15 & 12 & 4 & 3 & 2 \\
\hline
\end{tabular}

Table 3. Simple Visual Reaction Test, Reaction times (RT), motor reaction times (MT) and correct responses (CR)

\begin{tabular}{|l|l|l|l|}
\hline & RT(ms) & MT(ms) & CR \\
\hline PP (18) & $249.27 \pm 27.58$ & $105.08 \pm 24.97$ & $28.00 \pm 0.00^{*}$ \\
\hline NPP (20) & $252.14 \pm 37.89$ & $112.08 \pm 32.02$ & $27.45 \pm 0.99^{*}$ \\
\hline TS (35) & $256.72 \pm 34.60$ & $121.80 \pm 32.11$ & $27.80 \pm 0.47$ \\
\hline
\end{tabular}

${ }^{*} p<.05$

$\mathrm{PP}=$ professional players; $\mathrm{NPP}=$ non-professional players, $\mathrm{TS}=$ traditional sportspeople

Table 4 represents the data for simple acoustic test.

Those results did not differ statistically significant between the three groups. On average, the professional players again showed the shortest reaction and motor reaction times and the highest number of correct responses.

Table 5 represents results for the choice reaction test. Since in this test only 16 out of 50 stimuli required a reaction, the table includes an additional column for false responses.

As in the acoustic reaction test, the data did not differ significantly between the groups. This time and on average, the non-professional players showed the shortest reaction time and the smallest number of false responses. Professional players again displayed the shortest motor reaction times, traditional sportspeople the most correct responses.

Looking at the results for all three reaction tests (Table 3 to 5), each group indicated the significantly lowest reaction time in the simple acoustic test (Table 4). Thus, these results are comparable to other studies showing that people can react faster to acoustic signals than to visual ones (Chan \& Ng, 2012). The reaction times of the simple visual test (Table 3) were also significantly shorter than the reaction times of the choice reaction test (Table 5). As a result, the more complex test for choice reaction requires significantly longer reaction times than both simple reaction tests.

\section{Reaction and Motor Reaction Times in Different Game Genres}

As a further step, the results of the professional and non-professional eSports players were analyzed in terms of their game genre. For the game genre analysis, professional and non-professional players

Table 4. Simple Acoustic Reaction Test, Reaction times (RT), motor reaction times (MT) and correct responses (CR)

\begin{tabular}{|l|l|l|l|}
\hline & RT(ms) & MT(ms) & CR \\
\hline PP (18) & $208.41 \pm 27.83$ & $101.84 \pm 22.09$ & $27.94 \pm 0.24$ \\
\hline NPP (20) & $217.02 \pm 34.53$ & $108.02 \pm 21.61$ & $27.75 \pm 0.56$ \\
\hline TS (35) & $216.29 \pm 28.50$ & $112.23 \pm 22.54$ & $27.89 \pm 0.40$ \\
\hline
\end{tabular}

${ }^{*} \mathrm{p}<.05$

$\mathrm{PP}=$ professional players NPP = non-professional players, $\mathrm{TS}=$ traditional sportspeople 
Table 5. Choice Reaction Test, Reaction times (RT), motor reaction times (MT), correct responses (CR), and false responses (FR)

\begin{tabular}{|l|l|l|l|l|}
\hline & RT(ms) & MT(ms) & CR & FR \\
\hline PP (17) & $478.35 \pm 72.76$ & $111.22 \pm 30.57$ & $15.88 \pm 0.33$ & $0.06 \pm 0.24$ \\
\hline NPP (19) & $476.69 \pm 66.68$ & $116.06 \pm 24.13$ & $15.95 \pm 0.23$ & $0.05 \pm 0.23$ \\
\hline TS (34) & $482.68 \pm 57.93$ & $120.29 \pm 34.65$ & $15.97 \pm 0.17$ & $0.09 \pm 0.29$ \\
\hline
\end{tabular}

${ }^{*} p<.05$

$\mathrm{PP}=$ professional players; NPP = non-professional players, $\mathrm{TS}=$ traditional sportspeople

were not analyzed separately, because no differences in reaction and motor reaction times between these two groups were found. Table 6 represents results for the reaction tests.

ANOVAs with the independent variable game genre revealed significant differences in reaction times between the groups in all three tests $(\mathrm{RT} 1: \mathrm{F}(2,35)=4.10, \mathrm{p}<.05, \mathrm{f}=.19$; $\mathrm{RT} 2$ : $\mathrm{F}(2,35)=3.88$, $\mathrm{p}<.05, \mathrm{f}=.18$; RT3: $\mathrm{F}(2,33)=4.64, \mathrm{p}<.05, \mathrm{f}=.22)$. Post hoc tests confirmed these results only for RT2 ( $\mathrm{p}=.03)$ and RT3 ( $\mathrm{p}=.04)$. In these tests, sports simulation players showed significantly shorter reaction times than MOBA players.

Regarding motor reaction times, significant differences between the groups were found in the acoustic reaction test $(\mathrm{F}(2,35)=3.74, \mathrm{p}<.05, \mathrm{f}=.16)$. Post hoc tests revealed shorter reaction times regarding motor skills for sports simulation players compared to MOBA players $(\mathrm{p}<.05)$. Correct responses and false responses (only in the choice reaction test) did not differ between players from different game genres.

\section{Correlations for Reaction Times of All Tests}

Professional and non-professional eSports players did not reveal significant correlations between age, hours of sports per week, eSports per week or reaction times. Furthermore, ANOVA controlled for the variable age showed no significant influence on the performance in any of the three reaction tests. Additionally, their eSports experience in years did not correlate with any other results, while there was no association between age, hours of sports per week, or sports experience (in years), and reaction times for traditional sportspeople. Professional, non-professional players, and traditional sportspeople displayed strong positive correlations between reaction times over all three tests $($ all $\mathrm{p}<.05, \mathrm{r}>.50)$.

Table 6. Reaction (RT) and motor reaction time (MT) in simple visual (RT1), simple acoustic (RT2) and choice reaction test (RT3)

\begin{tabular}{|l|l|l|l|l|l|l|}
\hline & RT1 $(\mathbf{m s})$ & RT2(ms) & RT3(ms) & MT1(ms) & MT2(ms) & MT3(ms) \\
\hline MOBA (15) & $268.12 \pm 37.04$ & $\begin{array}{l}229.24 \pm \\
36.14^{*}\end{array}$ & $\begin{array}{l}516.49 \pm \\
69.67^{*}\end{array}$ & $\begin{array}{l}116.68 \pm \\
20.75\end{array}$ & $\begin{array}{l}116.25 \pm \\
20.12^{*}\end{array}$ & $\begin{array}{l}122.83 \pm \\
26.69\end{array}$ \\
\hline $\begin{array}{l}\text { First Person } \\
\text { Shooter (6) }\end{array}$ & $234.59 \pm 23.24$ & $204.67 \pm$ & $440.01 \pm$ & $118.28 \pm$ & $98.32 \pm$ & $117.40 \pm$ \\
& & 19.13 & 61.26 & 28.68 & 11.41 & 23.86 \\
\hline $\begin{array}{l}\text { Sports } \\
\text { simulation (17) }\end{array}$ & $241.19 \pm 25.89$ & $201.48 \pm$ & $457.38 \pm$ & $98.42 \pm$ & $97.64 \pm$ & $104.49 \pm$ \\
2 & & $24.71^{*}$ & $55.80^{*}$ & 21.92 & $22.54^{*}$ & 26.99 \\
\hline
\end{tabular}

${ }^{*} p<.05$ 


\section{DISCUSSION}

\section{Summary and Key Results}

The aim of this study was to compare the performance of professional to non-professional eSports players and traditional sportspeople in different reaction tests (visual, acoustic, choice reaction). No disparities were found between the three groups regarding reaction times and motor reaction times. Professional players were the only group without incorrect responses in the visual reaction test. Each group showed the lowest reaction and motor reaction times in the acoustic reaction test.

When comparing the eSports players regarding their game genres, differences in reaction times were found in all three tests. Especially in the acoustic and the choice reaction test, players from sports simulations reacted significantly faster than MOBA players.

\section{Participants Show Comparable Results for Reaction Times}

As a further step in this study, participants' data was compared to a standard sample provided by the manufacturer of the Vienna Test System. The standard sample (people from 16 to 35 years) provides comparable data given in 21 percentiles. The age range of the standard sample is similar to the participants' age (17 to 31 years), but there is no further information on the exact age distribution in the comparative data. Since the performance in reaction tests can decrease with increasing age (Dykiert et al., 2012), the comparisons between the data should be interpreted with caution. Professional, non-professional eSports players, and traditional sportspeople showed shorter reaction times in all three tests compared to most of the standard sample. For the simple visual reaction test $(\mathrm{N}=119,59$ females), the average reaction time on the medium percentile was at $273 \mathrm{~ms}$. For the simple acoustic reaction test ( $\mathrm{N}=101,47$ females) the medium percentile was at $228 \mathrm{~ms}$ and for the choice reaction test $(\mathrm{N}=106,55$ females) $557 \mathrm{~ms}$. The groups tested in our study displayed shorter reaction times on an average, than $60 \%$ of people from the standard sample in the visual, $70 \%$ of people in the acoustic and $85 \%$ in the choice reaction test. Compared to other studies using the Vienna Test System, average reaction times do not differ from those of volleyball players in the visual test and are shorter compared to non-sportspeople. For the choice reaction test, reaction times of professional, non-professional players, and traditional sportspeople were longer compared to professional fencers and to secondclass fencers (Johne et al., 2013; Zwierko et al., 2010).

High levels of perceptual and quick decision-making skills are essential in different sports (Pojskic et al., 2019) and eSports (Happonen \& Minashkina, 2019; Himmelstein et al., 2017) in order to perform successfully. These mental processes affect reaction times along with other factors, for instance afferent nerve conduction time or device response time (Badau et al., 2018). Additionally, eSports players need distinctive fine motor skills to achieve a high performance (Jenny et al., 2017) and often show better motor skills than non-gamers (Pluss et al., 2020). Likewise, physical activity can improve motor skills (Statton et al., 2015). The lack of differences between professional, nonprofessional eSports players, and traditional sportspeople in our study therefore suggests that sports and eSports may be the underlying factors to improve reaction and motor reaction times to a similar extent.

\section{No Differences in Motor Reaction Times}

Motor reaction times of participants in this study were slightly faster than $50 \%$ of the standard sample for visual reaction $(123 \mathrm{~ms})$ and acoustic reaction $(114 \mathrm{~ms})$. For the choice reaction, participants in our study performed better than the standard sample (medium percentile $149 \mathrm{~ms}$ ). Therefore, motor reaction times of traditional sportspeople, professional, and non-professional eSports players in this test were slightly better on an average compared to most of the normal population.

In similar studies, sportspeople generally showed comparable motor reaction times in visual reaction tests as professional eSports players, while non-sportspeople results were comparable to sportspeople in our study (Zwierko et al., 2010), but motor reaction times in choice reaction tests of professional and non-professional fencers (Johne et al., 2013) were shorter. Especially in sports such 
as fencing, fast and precise movements are very important and professional fencers outperformed participants in our study.

Professional, non-professional eSports players, and traditional sportspeople delivered faster results than the normal population. Compared to other sportspeople, however, all three groups showed comparable results to other non-professional sportspeople (e.g., volleyball) and were outperformed by professional sportspeople (e.g., fencers) in both parameters.

\section{High Correlations Between Reaction and Motor Reaction Times}

Correct responses differed significantly between professional and non-professional players in the visual reaction test. The incomplete responses result from missing the reaction key in the test situation with high pressure of time. Therefore, this is a case of both motor and cognitive failure. However, the operating tool has only limited similarity with a keyboard or mouse and certainly not with a game controller. Therefore, the results are hardly transferable to real situations in eSports and no further statement can be made regarding motor skills under time pressure of professional and non-professional eSports players in practice.

No correlations could be found between reaction and motor reaction times and other descriptive variables in all three tests for any of the groups. For the traditional sportspeople, correlations were analyzed for age and duration of sports per week, for professional and non-professional eSports players, correlations were calculated for age, eSports experience, and hours of playtime per week. Since there were no correlations found, it is impossible to make a statement on the link between the results and duration of sports or eSports per week. Additionally, it is unclear if a particular duration may improve reaction times most effectively. For future studies with more participants, correlations between performance and age may be of particular interest, as age can certainly influence the performance in reaction tests (Dykiert et al., 2012). Just like other studies on reaction times (Atan \& Akyol, 2014), reaction and motor reaction times are strongly correlated with all participants in the different tests. Participants with short reaction times often had quick motor skills available and vice versa.

\section{Players from Sports Simulation React Faster Than MOBA Players}

The following paragraphs provide an overview for the results of the professional and non-professional eSports players regarding their game genre. The results of reaction times among players from the three genres were different in all tests administered. Especially in acoustic and choice reaction test, players from sports simulations outperformed MOBA players significantly. Regarding descriptive mean values, FPS players, all played Counter-Strike: Global Offensive (Valve Corporation, 2012), had the shortest reaction times in the visual and choice reaction tests. However, the number of players in this group was smaller, which may be the reason for non-significant results. Studies have repeatedly shown that especially players of action video games, like FPS or Fighting Games, have shorter reaction times than non-gamers (Dye et al., 2009). Playing action video games is linked with enhanced attentional abilities (Dale et al., 2020), better visual searching (Wu \& Spence, 2013), oculomotor abilities (Chisholm \& Kingstone, 2012), and distribute visual attention over space (Qiu et al., 2018). It is therefore not surprising that especially FPS players had the shortest average reaction times in two tests.

However, on average, sports simulation players showed even shorter reaction times than FPS players in the acoustic test and clearly outperformed MOBA players in acoustic and choice reaction tests. Apart from action video games mentioned above, there is very little research available on cognitive abilities of players from different game genres. The strict classification as action video games and non-action video games of about 15 years ago is no longer appropriate today, as many other and new game genres have adopted features from action video games and vice versa (Dale et al., 2020). Important abilities for quick reactions like attentional capabilities or visual search strategies are also important in games like MOBAs or sports simulations.

Nevertheless, it is noteworthy that sports simulation players reacted significantly faster than the MOBA players. Both groups clearly did not differ in video game time or sports per week, eSports 
experience, or age. Assumptions can only be made regarding specific reasons for these results. Only one sports simulation player did not play a soccer simulation on PlayStation 4 (Sony Electronics \& Foxconn, 2013) or Xbox One (Flextronics \& Foxconn, 2013) (all but one played FIFA 19; one played MADDEN NFL 19 (EA Tiburon, 2018)), MOBA players all participated in League of Legends. Visual attention is essential in both genres, while acoustic attention should be more important in MOBAs. Therefore, there are no obvious reasons explaining why the players in this study show better results for reaction times. For more information about reaction times of players from different game genres, the number of participants should be increased, and all game genres (including RTS and Fighting Games) should be covered.

In one of the few studies that compared different abilities of players from MOBA, FPS, and MMORPG between game genres, Deleuze et al. (2017) found that FPS players showed significantly shorter simple reaction times than MOBA players and non-significantly $(\mathrm{p}=.07)$ shorter times than MMORPG players. Also, MOBA players comparatively took the longest time to react. This might indicate a connection between specific games and certain cognitive abilities, which should be investigated in future studies.

Additionally, results of Li et al. (2020) suggest that players of different levels vary in regard to specific cognitive abilities. In their study, top MOBA players outperformed lower ranked players in two cognitive tests (task-switching paradigm, go/no-go test). Both groups had similar gaming experience and playing time per week. The authors therefore assumed that certain cognitive skills are not related to a player's gaming experience, but instead to the level of performance. Similar to our study, eSports players in future studies should thus be divided according to game genre and performance level. Gaming experience and playtime per week only seem to play a minor role regarding certain cognitive abilities, which may affect the players' reaction times.

\section{Strengths and Limitations}

Previous studies on video game reaction times selected players based primarily upon their selfreported play time or experience via questionnaires. Players often overestimate or underestimate their gaming behavior or play different games (for an overview regarding self-reported game time via questionnaires see Dale et al., 2020). The present paper is one of the first studies which compares gamers and their actual observed performance level in different games. Especially professional players invest considerable time in games and are left with hardly any time for other games. Therefore, the influence of other games on cognitive abilities may be lower in these participants than in the mostly non-professional gamers from other studies.

The group of players in the present study reflected the most popular genres in eSports (Bányai et al., 2019), except for the RTS and Fighting Games. In addition to FPS, quick reaction times are of great importance, especially in RTS and Fighting game genres. Future studies should therefore also cover those two genres. Furthermore, participants' distribution in the three examined genres must be regarded as a weakness. The eSports group contained less FPS players than MOBA or sports simulation players, which showed the shortest reaction times in visual and choice reaction tests (Table 6). However, these differences were not statistically significant, which may be due to the group sizes. Larger samples from different genres are required in order to compare abilities like reaction times of eSports players from different game genres. Only long-term intervention studies can tell whether games are helpful in improving skills such as the reaction time, or whether a player chooses a specific game because they already have good cognitive abilities at hand (Dale et al., 2020). Such issues should be addressed in studies on performance indicators in eSports.

Within this study traditional sportspeople were divided into different groups regarding their main form of sports. Statistical analyses showed no differences between traditional sports from disciplines with a high demand in reaction time (e.g., short sprints, combat sports), team sports (e.g., soccer, basketball), or disciplines without specific demands on reaction times (e.g., endurance sports like running). Moreover, it must be considered that the sportspeople group in this study 
were non-professionals and therefore there was no information on the actual performance level. A separate control group (non-gamers and non-sportspeople) should also be tested in future studies. The results of an actual control group would carry more meaning than the standard sample given by the manufacturer of the Vienna Test System.

This study also recorded how many hours of traditional sport the eSports player did on average per week. However, the type of sport was not taken into account. For instance, an eSports player might do traditional sports like martial arts, which also requires fast reaction times. Therefore, the influence of traditional sports on the eSports players' reaction times cannot be further investigated in this study. This needs to be considered as a weakness.

\section{CONCLUSION}

Many physical and cognitive factors wield influence on general reaction time (Badau et al., 2018). However, these numerous factors can hardly be influenced or considered in a study like this. Therefore, this study attempts to draw first comparisons between the reaction times of traditional sportspeople and eSports players. This research is intended to create an impression of whether further research on reaction time - a possible performance factor in eSports - can be conducted in the future. As a result of this study, traditional sportspeople, professional, and non-professional eSports players showed no differences in reaction and motor reaction times in three different tests (simple visual, simple acoustic, and choice reaction test). Additionally, each group showed generally shorter reaction times than the Vienna Test System standard sample. These results may indicate that sports and eSports can improve overall reaction times to a similar extent. However, the comparative data stem from the Vienna Test System publisher, not a control group specific for this study. To strengthen the validity of these results, a control group should be tested under the same conditions for future studies. In addition to a larger test sample, not only the eSports players but also traditional sportspeople should be specifically subdivided according to type of sports and performance level, in order to further increase the data. The precise form of sports played by the eSports players should also be included in future studies. This way, links between game genre and type of sports can be determined, for instance if a FIFA 19 or Pro Evolution Soccer 2019 (PES Productions, 2018) player also plays soccer in their free time.

The same also applies to future tests researching the difference between game genres. As conducted in earlier studies (Deleuze et al., 2017), MOBA players showed the longest reaction times in this study, especially compared to the sports simulation players. Therefore, different games may affect reaction times to a different extent. These differences can represent goals of future research. Why it was particularly MOBA players, who reacted the slowest, is not yet determined. Objectively speaking, fast reactions in MOBA games are as essential as in other eSports titles. In the future, it will also be necessary to consider other influencing factors such as age, sports and the performance level.

While the eSports market grew rapidly in the last years, there is still little knowledge about performance factors of eSports players (Pedraza-Ramirez et al., 2020). This makes further research in this field an essential requirement. The ability to react quickly is an important factor in eSports. Although this is influenced by many parameters, it can serve as a first approach to detect differences between the performance of eSports players. In their review, Pedraza-Ramirez et al. (2020) listed different cognitive factors such as working memory or cognitive flexibility, which also may influence the performance in eSports. Furthermore, motoric skills (Donaldson, 2015) play an important role in eSports performance as well. These cognitive and tactile skills could also form the basis for training of eSports players, away from their computer or gaming console. Since there are already training courses for eSports existing, a scientific basis serves as a crucial foundation for the contents taught there. In addition, as in traditional sports, scouting could take place in eSports based on specific performance parameters. Of course, it must be considered that understanding of the game and talent for the game also greatly influence the player's performance and these parameters are very difficult to investigate in future research. 


\section{ACKNOWLEDGMENT}

This research was supported by the AOK Rheinland/Hamburg.

We wish to thank Marius Loewe and Markus Soffner for their assistance in data collections and all participants for their contribution. We also want to thank the AOK Rheinland/Hamburg for their support. 


\section{REFERENCES}

Atan, T., \& Akyol, P. (2014). Reaction Times Of Different Branch Athletes And Correlation Between Reaction Time Parameters. Procedia: Social and Behavioral Sciences, 116, 2886-2889. doi:10.1016/j.sbspro.2014.01.674

Badau, D., Baydil, B., \& Badau, A. (2018). Differences Among Three Measures Of Reaction Time Based On Hand Laterality In Individual Sports. Sports (Basel, Switzerland), 6(2), 45. doi:10.3390/sports6020045 PMID:29910349

Bányai, F., Griffiths, M. D., Király, O., \& Demetrovics, Z. (2019). The Psychology Of Esports: A Systematic Literature Review. Journal of Gambling Studies, 35(2), 351-365. doi:10.1007/s10899-018-9763-1 PMID:29508260

Braun, B., Stopfer, J. M., Müller, K. W., Beutel, M. E., \& Egloff, B. (2016). Personality And Video Gaming: Comparing Regular Gamers, Non-Gamers, And Gaming Addicts And Differentiating Between Game Genres. Computers in Human Behavior, 55, 406-412. doi:10.1016/j.chb.2015.09.041

Chan, A. H. S., \& Ng, A. W. Y. (2012). Finger Response Times To Visual, Auditory And Tactile Modality Stimuli: Lecture Notes In Engineering And Computer Science. Newswood Limited. Http://Www.Scopus.Com/Inward/ Record.Url?Scp=84867462382\&Partnerid=8yflogxk

Chen, R., Chen, J., \& Li, L. (2015). Action Videogame Play Improves Visual Motor Control. Journal of Vision (Charlottesville, Va.), 15(12), 42. doi:10.1167/15.12.42

Chisholm, J. D., \& Kingstone, A. (2012). Improved Top-Down Control Reduces Oculomotor Capture: The Case Of Action Video Game Players. Attention, Perception \& Psychophysics, 74(2), 257-262. doi:10.3758/ s13414-011-0253-0 PMID:22160821

Dale, G., Joessel, A., Bavelier, D., \& Green, C. S. (2020). A New Look At The Cognitive Neuroscience Of Video Game Play. Annals of the New York Academy of Sciences, 1464(1), 192-203. doi:10.1111/nyas.14295 PMID:31943260

Damato, B., \& Groenwald, C. (2003). Multifixation Campimetry On Line [Computer Software]. St Paul's Eye Unit, Royal Liverpool University Hospital. Https://Bjo.Bmj.Com/Content/87/10/1296

Deleuze, J., Christiaens, M., Nuyens, F., \& Billieux, J. (2017). Shoot At First Sight! First Person Shooter Players Display Reduced Reaction Time And Compromised Inhibitory Control In Comparison To Other Video Game Players. Computers in Human Behavior, 72, 570-576. doi:10.1016/j.chb.2017.02.027

Donaldson, S. (2015). Mechanics And Metagame: Exploring Binary Expertise In League Of Legends. Games and Culture, 12(5), 426-444. doi:10.1177/1555412015590063

Dye, M. W. G., Green, C. S., \& Bavelier, D. (2009). Increasing Speed Of Processing With Action Video Games. Current Directions in Psychological Science, 18(6), 321-326. doi:10.1111/j.1467-8721.2009.01660.x PMID:20485453

Dykiert, D., Der, G., Starr, J. M., \& Deary, I. J. (2012). Age Differences In Intra-Individual Variability In Simple And Choice Reaction Time: Systematic Review And Meta-Analysis. PLoS One, 7(10), E45759. doi:10.1371/ journal.pone.0045759 PMID:23071524

EA Tiburon. (2018). Madden Nfl 19 [Video Game]. EA Sports. Https://Www.Ea.Com/Games/Madden-Nfl/ Madden-Nfl-19

EA Vancouver. (2018). Fifa 19 [Video Game]. EA Sports. Https://Www.Ea.Com/Games/Fifa/Fifa-19

Eyesage.Org. (2014). Eyescream Software Ii (Version 2.3.1) [Computer Software]. Eyesage.Org. Http://Www. Eyesage.Org/?Lang=Us

Feng, J., Spence, I., \& Pratt, J. (2007). Playing An Action Video Game Reduces Gender Differences In Spatial Cognition. Psychological Science, 18(10), 850-855. doi:10.1111/j.1467-9280.2007.01990.x PMID:17894600

Flextronics \& Foxconn. (2013). Xbox One [Video Gaming Console]. Microsoft. Https://Www.Xbox.Com/

Ghuntla, T., Gokhale, P., Mehta, H., \& Shah, C. (2014). A Comparison And Importance Of Auditory And Visual Reaction Time In Basketball Players. Saudi Journal Of Sports Medicine, 14(1), 35. Https://Doi.Org/10.4103/13196308.131616 
Green, C. S., \& Bavelier, D. (2003). Action Video Game Modifies Visual Selective Attention. Nature, 423(6939), 534-537. doi:10.1038/nature01647 PMID:12774121

Green, C. S., Li, R., \& Bavelier, D. (2010). Perceptual Learning During Action Video Game Playing. Topics in Cognitive Science, 2(2), 202-216. doi:10.1111/j.1756-8765.2009.01054.x PMID:25163784

Green, W. (2018, June 20). Sport Management \& Sport Psychology For Esport: Winning Championships. Https:// Medium.Com/@Mindgamesweldon/Sport-Management-Sport-Psychology-For-Esport-Winning-Championships717491 ba4609

Happonen, A., \& Minashkina, D. (2019). Professionalism In Esport: Benefits In Skills And Health \& Possible Downsides. Https://Doi.Org/10.13140/Rg.2.2.28958.59208

Himmelstein, D., Liu, Y., \& Shapiro, J. L. (2017). An Exploration Of Mental Skills Among Competitive League Of Legend Players. International Journal of Gaming and Computer-Mediated Simulations, 9(2), 1-21. doi:10.4018/IJGCMS.2017040101

Hodge, V., Devlin, S., Sephton, N., Block, F., Cowling, P., \& Drachen, A. (2019). Win Prediction In MultiPlayer Esports: Live Professional Match Prediction. IEEE Transactions on Games, 1. Https://Doi.Org/10.1109/ Tg.2019.2948469

Hüttermann, S., Ford, P. R., Williams, A. M., Varga, M., \& Smeeton, N. J. (2019). Attention, Perception, And Action In A Simulated Decision-Making Task. Journal of Sport \& Exercise Psychology, 41(4), $230-241$. doi:10.1123/jsep.2018-0177 PMID:31319400

IBM. (2019). IBM SPSS Statistics 26 [Computer Software]. IBM.

Jenny, S. E., Manning, R. D., Keiper, M. C., \& Olrich, T. W. (2017). Virtual(Ly) Athletes: Where Esports Fit Within The Definition Of "Sport”. Quest, 69(1), 1-18. doi:10.1080/00336297.2016.1144517

Johne, M., Poliszczuk, T., Poliszczuk, D., \& Da̧browska-Perzyna, A. (2013). Asymmetry Of Complex Reaction Time In Female Épée Fencers Of Different Sports Classes. Polish Journal of Sport and Tourism, 20(1), 25-29. doi:10.2478/pjst-2013-0003

Kowal, M., Toth, A. J., Exton, C., \& Campbell, M. J. (2018). Different Cognitive Abilities Displayed By Action Video Gamers And Non-Gamers. Computers in Human Behavior, 88, 255-262. doi:10.1016/j.chb.2018.07.010

Li, X., Huang, L., Li, B., Wang, H., \& Han, C. (2020). Time For A True Display Of Skill: Top Players In League Of Legends Have Better Executive Control. Acta Psychologica, 204, 103007. doi:10.1016/j.actpsy.2020.103007 PMID:32000064

Lipovaya, V., Lima, Y., Grillo, P., Barbosa, C. E., Souza, J. M. De, \& Duarte, F. J. De C. M. (2018). Coordination, Communication, And Competition In Esports: A Comparative Analysis Of Teams In Two Action Games. Proceedings Of $16^{\text {th }}$ European Conference On Computer-Supported Cooperative Work - Exploratory Papers. Https://Doi.Org/10.18420/Ecscw2018_11

Mangeloja, E. (2019). Economics Of Esports. Electronic Journal Of Business Ethics And Organization Studies, 24(2), 34-42.

Newzoo. (2020). Newzoo Global Esports Market Report 2020: Light Version. Https://Newzoo.Com/Insights/ Trend-Reports/Newzoo-Global-Esports-Market-Report-2020-Light-Version/

Paradisis, G. (2013). Association Of Reaction Time And Performance In 60 And 100 M. New Studies In Athletics, (28), 95-103.

Parshakov, P., Coates, D., \& Zavertiaeva, M. (2018). Is Diversity Good Or Bad? Evidence From Esports Teams Analysis. Applied Economics, 50(47), 5064-5075. doi:10.1080/00036846.2018.1470315

Pedraza-Ramirez, I., Musculus, L., Raab, M., \& Laborde, S. (2020). Setting The Scientific Stage For Esports Psychology: A Systematic Review. International Review of Sport and Exercise Psychology, 6(3), 1-34. doi:10 $.1080 / 1750984 X .2020 .1723122$

Pes Productions. (2018). Pro Evolution Soccer 2019 [Video Game]. Konami. Https://Www.Konami.Com/ Wepes/2019/ 
Pluss, M. A., Novak, A. R., Bennet, K. J., Panchuk, D., Coutts, A. J., \& Fransen, J. (2020). Perceptual-Motor Abilities Underlying Expertise In Esports. Journal of Expertise, 2(3).

Pojskic, H., Pagaduan, J., Uzicanin, E., Separovic, V., Spasic, M., Foretic, N., \& Sekulic, D. (2019). Reliability, Validity And Usefulness Of A New Response Time Test For Agility-Based Sports: A Simple Vs. Complex Motor Task. Journal of Sports Science \& Medicine, 18(4), 623-635. PMID:31827346

Qiu, N., Ma, W., Fan, X., Zhang, Y., Li, Y., Yan, Y., Zhou, Z., Li, F., Gong, D., \& Yao, D. (2018). Rapid Improvement In Visual Selective Attention Related To Action Video Gaming Experience. Frontiers in Human Neuroscience, 12, 47. doi:10.3389/fnhum.2018.00047 PMID:29487514

Richardson, B., Ellis, D., Greenwald, R. C. A., Cherry, J., \& Meador, C. (2014). Reaction Times Differences In Video Game And Non Video Game Players [Poster]. Central Washington University. Https://Digitalcommons. Cwu.Edu/Cgi/Viewcontent.Cgi?Article $=1689 \&$ Context $=$ Source

Riot Games. (2009). League Of Legends [Video Game]. Riot Games. Https://Na.Leagueoflegends.Com/

Schubert, T., Finke, K., Redel, P., Kluckow, S., Müller, H., \& Strobach, T. (2015). Video Game Experience And Its Influence On Visual Attention Parameters: An Investigation Using The Framework Of The Theory Of Visual Attention (Tva). Acta Psychologica, 157, 200-214. doi:10.1016/j.actpsy.2015.03.005 PMID:25834984

Schuhfried, G., \& Prieler, J. (1997). Vienna Test System [Computer Software]. Schuhfried. Https://Www. Schuhfried.Com/Vienna-Test-System/

Sony Electronics \& Foxconn. (2013). Playstation 4 [Video Gaming Console]. Sony Computer Entertainment. Https://Www.Playstation.Com/Ps4/?Smcid=Pdc\%3ade-De\%3aprimary\%20nav\%3amsg-Hardware\%3aps4

Statton, M. A., Encarnacion, M., Celnik, P., \& Bastian, A. J. (2015). A Single Bout Of Moderate Aerobic Exercise Improves Motor Skill Acquisition. PLoS One, 10(10), E0141393. doi:10.1371/journal.pone.0141393 PMID:26506413

Tabachnick, B. G., \& Fidell, L. S. (2010). Using Multivariate Statistics (5th ed.). Pearson/Allyn And Bacon.

Valve Corporation. (2012). Counter-Strike: Global Offensive [Video Game]. Valve Corporation. Https://Store. Steampowered.Com/App/730/Counterstrike_Global_Offensive/

Voss, M. W., Kramer, A. F., Basak, C., Prakash, R. S., \& Roberts, B. (2010). Are Expert Athletes 'Expert' In The Cognitive Laboratory? A Meta-Analytic Review Of Cognition And Sport Expertise. Advance Online Publication. Applied Cognitive Psychology, 24(6), 812-826. Https://Doi.Org/10.1002/Acp.1588

Wagner, M. G. (2006). On The Scientific Relevance Of Esports. International Conference On Internet Computing, 437-442.

Wu, S., \& Spence, I. (2013). Playing Shooter And Driving Videogames Improves Top-Down Guidance In Visual Search. Attention, Perception \& Psychophysics, 75(4), 673-686. doi:10.3758/s13414-013-0440-2 PMID:23460295

Zwierko, T., Osinski, W., Lubinski, W., Czepita, D., \& Florkiewicz, B. (2010). Speed Of Visual Sensorimotor Processes And Conductivity Of Visual Pathway In Volleyball Players. Journal of Human Kinetics, 23(1). Advance online publication. doi:10.2478/v10078-010-0003-8 
Peter Bickmann is a staff member and research associate in the eSports project at the German Sport University Cologne. He records and analyzes possible performance parameters like eye-tracking data or reaction times of differently skilled eSports players and investigates new approaches for the usage of eye-tracking as a training tool in eSports.

Konstantin Wechsler $(P h D)$ is a staff member and research associate in the eSports project at the German Sport University Cologne. His main tasks include the management of eSport performance tests and the design of cognitive testing for eSports players.

Kevin Rudolf $(P h D)$ is a staff member and research associate in the eSports project at the German Sport University Cologne. His lines of action are the planning and evaluation of eSport survey studies.

Chuck Tholl is a staff member and research associate in the eSports project and doctoral candidate at the German Sport University Cologne. He functions as an instructor for eSports performance tests and adapts classical therapy concepts for eSports.

Ingo Froböse (PhD) is head of the Institute of Movement Therapy and Movement-oriented Prevention and Rehabilitation at the German Sport University Cologne. Professor Froböse navigates the eSports project at the University. As a lateral thinker in science, he founded the eSports project.

Christopher Grieben $(P h D)$ is a research associate in the Institute of Health Promotion and Clinical Movement Science at the German Sport University Cologne. He focuses on research and teaching with regard to the impact of physical activity and sport on health all the way to questions of health communication, ergonomics and product testing. He also chairs the eSports working group at the German Sport University Cologne. 